American Journal of Pharmacology and Toxicology 3 (1): 125-136, 2008

ISSN 1557-4962

(C) 2008 Science Publications

\title{
On the Hormetic Behaviour of Drugs Binding to Different Redox States of Amine Oxidase Enzymes
}

\author{
${ }^{1}$ Deepak Narang, ${ }^{1}$ G. Reid McDonald, ${ }^{2}$ David J. Smith, \\ ${ }^{3}$ Maria Luisa Di Paolo, ${ }^{4}$ Dale E. Edmondson and ${ }^{1}$ Andrew Holt \\ ${ }^{1}$ Department of Pharmacology, Faculty of Medicine and Dentistry, \\ 9-70 Medical Sciences Building, University of Alberta, Edmonton, Alberta, Canada, T6G 2H7 \\ ${ }^{2}$ BioTie Therapies Corp., Tykistökatu 6, FIN-20520 Turku, Finland \\ ${ }^{3}$ Department of Biological Chemistry, \\ University of Padua, Viale G. Colombo 3, 35121 Padua, Italy \\ ${ }^{4}$ Department of Biochemistry, \\ Emory University School of Medicine, 1510 Clifton Rd., Atlanta, GA 30322, USA
}

\begin{abstract}
Classical monoamine oxidases and copper-containing semicarbazide-sensitive amine oxidases are drug targets for a variety of established and novel pharmaceuticals used to treat conditions such as depression, Parkinsonism and inflammatory disorders. Development of enzyme inhibitors, both historically and currently, assumes an underlying adherence of these enzymes to Michaelis-Menten kinetic principles. In this mini-review, we discuss historical data from several laboratories and novel data from our own laboratories which show clearly that such an assumption is invalid. Rather, these enzymes often display hormetic behaviour towards their substrates, resulting in bell-shaped kinetic plots. We outline possible underlying mechanisms which might account for this behaviour and show how novel reversible hormetic drugs may capitalise on these mechanisms to introduce a new dimension in selectivity. The potential future benefits for therapeutic modulation of amine oxidase activities are discussed.
\end{abstract}

Key words: enzyme kinetics, amine oxidases, substrates, imidazolines, cations

\section{INTRODUCTION}

Mammalian amine oxidase enzymes play critically important roles in physiological and pathological tissue development and function. These roles are, for the most part, directly related to the enzymatic capacity of these proteins to oxidise endogenous amines, ultimately yielding an aldehyde, an ammonium derivative and hydrogen peroxide.

Classical flavin-containing mitochondrial monoamine oxidase (MAO) enzymes (EC 1.4.3.4) are responsible for regulating vesicular concentrations of biogenic amine neurotransmitters in neurons, while hepatic and intestinal MAO isozymes serve to protect the host against deleterious effects of dietary amines ${ }^{[49]}$. Copper-containing Semicarbazide-Sensitive Amine Oxidase (SSAO) enzymes (EC 1.4.3.6) are found mainly on the plasma membranes of vascular smooth muscle cells and adipocytes ${ }^{[3,27]}$ and are expressed on vascular endothelium in some tissues following an inflammatory insult ${ }^{[42]}$. These enzymes act to tether lymphocytes to the vascular wall at sites of inflammation and to generate hydrogen peroxide $\left(\mathrm{H}_{2} \mathrm{O}_{2}\right)$ as a signaling molecule involved in inflammation $^{[18]}$, glucose metabolism ${ }^{[51]}$ and possibly vascular function ${ }^{[5]}$. Diamine oxidase (DAO), also a member of the EC 1.4.3.6 sub-subfamily, is found as an extracellular enzyme in various tissues, including intestine, where it functions to protect the host against ingested histamine ${ }^{[28]}$. Lysyl oxidase (LOX; EC 1.4.3.13) is an extracellular copper-containing enzyme which oxidizes the side chain of peptidyl lysine ${ }^{[25]}$. LOX-mediated generation of a peptidyl $\alpha$-aminoadipic$\delta$-semialdehyde side chain then facilitates cross-linking of collagen and elastin chains in the extracellular matrix $^{[25]}$. Several LOX-like proteins have recently been described which share numerous structural and functional similarities with LOX and at least one of

Corresponding Author: Andrew Holt, Department of Pharmacology, Faculty of Medicine and Dentistry, 9-70 Medical Sciences Building, University of Alberta, Edmonton, Alberta, Canada, T6G 2H7 Tel: 1-780-492-8620 Fax: 1-780-492-4325 
these is highly expressed in invasive cancer cells ${ }^{[1]}$. Finally, flavin-containing polyamine oxidase (EC 1.5.3.11) is a peroxisomal enzyme which oxidizes $\mathrm{N}$ acetylated polyamines and acts, in concert with other enzymes, to regulate levels of polyamines and their metabolites which are important in cellular growth ${ }^{[43]}$.

In humans, two EC 1.4.3.6 enzymes are also found as soluble proteins circulating in the blood. As well as DAO which increases in the later stages of pregnancy ${ }^{[9]}$, SSAO may be cleaved from its extracellular sites on the vascular wall and perhaps on adipocytes and can be detected at very low levels in the circulation. In contrast, ruminants have high levels of a circulating SSAO activity, secreted from the liver, which is structurally distinct from tissue-bound SSAO in those species ${ }^{[30]}$. Herein, we will refer to the circulating human enzyme cleaved from the vessel wall as human plasma SSAO and to the liver-derived enzyme in bovine plasma as bovine plasma amine oxidase (BPAO).

The important roles played by these enzymes in numerous processes have led to the development of drugs targeting specific enzyme sub-subfamilies, or even isozymes within a sub-subfamily. For example, phenelzine and tranylcypromine are potent irreversible inhibitors of MAO enzymes and their capacity to inhibit the MAO-A isozyme in particular means that these drugs are effective antidepressants ${ }^{[20]}$. Moclobemide is a competitive, reversible and selective MAO-A inhibitor possessing antidepressant efficacy, which is arguably safer for patients than the older irreversible inhibitors $^{[10]}$. Deprenyl (selegiline) is an irreversible MAO inhibitor which shows significant selectivity for MAO-B; deprenyl is thus useful in enhancing dopaminergic transmission in Parkinson's disease patients $^{[8]}$. Further neuroprotective properties of deprenyl unrelated to MAO-B inhibitory potency have also been described ${ }^{[22]}$. Inhibitors of SSAO enzymes may reduce glucose uptake into several cell types ${ }^{[50]}$, but inhibitor development is presently focusing upon the usefulness of such drugs in reducing lymphocyte rolling and adhesion and thus acting in an antiinflammatory manner, in damaged tissue ${ }^{[33]}$. In this regard, inhibition of SSAO-mediated hydrogen peroxide production appears to be responsible for the anti-inflammatory effect, since peroxide acts as a signaling molecule which stimulates expression of adhesion proteins on vascular endothelium ${ }^{[18]}$.

The success of industrial programs aimed at developing novel inhibitors of amine oxidases with improved selectivity and pharmacokinetic attributes relies very much upon a capability to quantify inhibitor effects in appropriate kinetic model systems. In turn, an in-depth understanding of the kinetic behaviour of amine oxidases towards a variety of substrates is vital if the effects of inhibitors are to be modeled and quantified correctly. While several researchers have contributed substantially to our understanding of molecular mechanisms associated with activities of MAO and SSAO in particular ${ }^{[15,23,31,32,37]}$, basic kinetic data ( $\mathrm{v}$ versus $[\mathrm{S}]$ in the absence and presence of established or novel reversible inhibitors) are usually analysed by applying the Michaelis-Menten equation:

$$
\mathrm{v}=\frac{\mathrm{V}_{\max }[\mathrm{S}]}{\mathrm{K}_{\mathrm{M}}+[\mathrm{S}]}
$$

This equation describes a rectangular hyperbola generated in a unireactant (one substrate) system. $\mathrm{V}_{\max }$ refers to the rate of substrate turnover when substrate is present at a concentration which ensures that the time for which the enzyme active site is unoccupied (between departure of product and binding of substrate) is as short as is physically possible. Since catalytic rate constants for amine oxidases are relatively slow, the Michaelis constant, $\mathrm{K}_{\mathrm{M}}$, approximates an equilibrium constant for substrate binding, thereby providing an indication of substrate affinity for the enzyme.

Several complications may be identified with regard to the practice of applying Michaelis-Menten kinetics to amine oxidase assay data. The most obvious of these is that amine oxidases do not catalyse unireactant reactions; with MAO and SSAO enzymes, reactions are bi-bi and bi-ter, respectively, involving two substrates (amine and dioxygen) and generating either two products (imine - which is hydrolysed nonenzymatically in solution yielding aldehyde and ammonium - and hydrogen peroxide with MAO) or three products (aldehyde, ammonium and hydrogen peroxide with SSAO). Nevertheless, if it is ensured that one substrate (dioxygen) is present at a constant (usually saturating) concentration, variations in the concentration of the other substrate (amine) should result in changes in initial velocity (v) which are related to [amine] through a rectangular hyperbolic, or Michaelis-Menten, relationship. Accordingly, any problems relating to the bireactant nature of these enzymes are avoided.

Unfortunately, when initial reaction velocities are determined for MAO and SSAO enzymes, it is not uncommon to observe rates lower than expected as these enzymes approach saturation with amine, resulting in bell-shaped hormetic curves when initial velocities are plotted versus [amine]. For example, oxidation of the MAO-B substrate, $\beta$-phenylethylamine 
(PEA), results in time-dependent inhibition of the enzyme, with this effect being more apparent at higher substrate concentrations, resulting in a bell-shaped kinetic curve ${ }^{[21]}$. It is not uncommon for such a reduction in the rate of product formation at higher amine concentrations, regardless of the identity of the enzyme or the amine substrate, to be dismissed as product inhibition, or as some other artefact, with the result that points are ignored, or assays are never done at amine concentrations high enough to generate these anomalous data. The resulting kinetic data may then be fitted quite successfully to rectangular hyperbolae with the Michaelis-Menten equation.

If assays are done carefully such that initial reaction velocities are measured, then concentrations of aldehyde (and other products) generated over an initial brief reaction period are negligible. This is particularly true when radiochemical or fluorescence-based assay systems are used, since the high sensitivity associated with these approaches means very low enzyme concentrations can be used and substantial signals can be detected following oxidation of only a tiny fraction of total substrate initially present. Under these circumstances, having taken steps to ensure that rates determined from discontinuous procedures represent initial velocities, hormetic $v$ versus $[\mathrm{S}]$ plots can not be explained by implicating product inhibition. Rather, such data suggest that amine substrate may bind in an incorrect conformation or to a second site on the enzyme protein, resulting in a complex which is catalytically incompetent, or at least which turns over substrate more slowly than would have been predicted from Michaelis-Menten analyses.

Observations of substrate inhibition are commonplace in many enzyme families. Indeed, it has been suggested that enzymes following classical Michaelis-Menten behaviour are far less common than those which display hormetic or otherwise anomalous kinetic plots ${ }^{[2]}$. For example, several hepatic cytochromes P450 involved in phase 1 drug oxidations give rise to hormetic kinetic plots and such data have been used to develop both a better understanding of P450 enzymatic mechanisms, as well as improved models and equations to analyse experimental data ${ }^{[45,46]}$. In particular, such studies reveal the degree to which kinetic constants may be incorrectly estimated on fitting Michaelis-Menten hyperbolae to the early (pseudohyperbolic) portion of bell-shaped data. Development of novel enzyme inhibitors as pharmaceuticals relies upon appropriate analysis of inhibitor kinetic data in order that inhibitor potencies and mechanisms may be identified and quantified correctly. With regard to amine oxidases, while several reversible inhibitors of
MAO and SSAO enzymes already exist, or are in preclinical stages of development, these are routinely assessed in kinetic studies which assume MichaelisMenten-like behaviour with respect to substrate oxidation. Yet, several published studies show clearly that such assumptions are inappropriate and that models and equations which account for hormetic behaviour towards substrates should be adopted. We outline below several published observations of hormetic SSAO behaviour, both from our laboratories and from other researchers, along with recent results which may offer an explanation for this phenomenon. We also discuss similar preliminary findings with human MAO and outline how the mechanisms leading to hormetic catalytic behaviour may offer novel targets for new compounds which themselves possess hormetic properties and which alter enzyme activities in a substrate-specific manner.

Anomalous kinetic behaviour of SSAO (EC 1.4.3.6) enzymes

Inhibition of SSAO enzymes at high substrate concentrations was first observed by McEwen in $1965^{[29]}$, in experiments where benzylamine (BZ) oxidation by an enzyme referred to as human plasma monoamine oxidase (i.e. plasma SSAO) had a maximal rate at around $300 \mu \mathrm{M}$ substrate, activity dropping markedly at lower or higher substrate concentrations. Lyles and Callingham ${ }^{[26]}$, in one of the earliest studies in which SSAO activity was measured and identified as being separate and distinct from classical MAO, showed that BZ oxidation by rat heart SSAO reached a peak and then fell at concentrations above $50 \mu \mathrm{M}, 5-$ fold higher than the $K_{M}(10 \mu M)$. These initial observations were confirmed in meticulous experiments with pig plasma SSAO, done by Bardsley and colleagues. With BZ as substrate, a concave EadieHofstee plot was obtained, indicating that at high substrate concentrations, activity was lower than would be predicted from Michaelis-Menten curve-fitting to early data points ${ }^{[2]}$. It was also proposed from initial kinetic data that $\mathrm{BZ}$ was able to bind to a second site on the enzyme, such that a ternary ESS complex was formed, resulting in altered turnover kinetics for BZ at the active site ${ }^{[2]}$. This contention was strengthened by further in-depth studies which indicated that hormetic behaviour towards substrate may result from interactions of BZ with more than one enzyme form, in a manner dependent upon $\mathrm{pH}$. Furthermore, enzyme activity versus a single substrate concentration could be modified by imidazole in a hormetic manner, with the extent of peak imidazole stimulation, as well as the concentration of imidazole at which peak activation occurred, also showing significant $\mathrm{pH}$ dependence ${ }^{[19]}$. 
More recently, Ignesti has examined hormetic behaviour displayed by several SSAO enzymes, including rat vascular SSAO and diamine oxidase from mammalian and plant sources ${ }^{[17]}$. Virtually every substrate examined showed bell-shaped kinetic plots and it was proposed that two substrate binding sites-one active site and one secondary site-must exist on each enzyme monomer in order to explain the observed kinetics. A series of models and equations was proposed, based on this assumption, which generally involved ordered substrate binding to a second binding site yielding a dead-end (non-productive) ESS complex. It was emphasised that use of Michaelis-Menten approximations at low substrate concentrations would be incorrect and that inhibitor terminology such as competitive, non-competitive and mixed, which are appropriate terms when used within a system complying with Michaelis-Menten kinetic principles, is incorrect and misleading when the enzyme of interest follows a more complex kinetic path. Similar hormetic substrate turnover has recently been demonstrated in SSAO from A. globiformis with PEA as substrate ${ }^{[44]}$. Interestingly, hormesis was only evident at $\mathrm{pH} 8.2$, as effects of the proposed dead-end ESS complex were not evident at $\mathrm{pH} 6.2$ or $7.2^{[44]}$. Whether hormetic behaviour is observed or not, and the degree to which it occurs, may also be dependent upon buffer ionic strength $^{[12]}$ and it is possible that observed hormetic enzyme behaviour is a consequence of reaction conditions in vitro rather than representing true in vivo behaviour. In this regard, care should be taken to minimise changes or differences in ionic strength in in vitro assays and it may be advisable for buffers to reflect the in vivo ionic environment of the enzyme ${ }^{[11]}$.

Our interest in examining the mechanism underlying non-hyperbolic substrate handling by SSAO enzymes arose as a result of the potential existence of a second site, binding of substrate to which apparently resulted in an inactive ternary ESS complex. Since the affinities of substrates for this second site appeared to be rather lower than their affinities for the active site, then drugs targeting this putative second site may offer the opportunity to inhibit SSAO in vivo while largely avoiding dosing problems associated with competition between substrate and inhibitor that occur when drugs target the enzyme active site. While neither the nature nor the location of this putative second site were known, Di Paolo and colleagues had demonstrated an ability of tetraphenylphosphonium to inhibit BPAO through binding to a site distinct from the active site ${ }^{[6]}$. Furthermore, numerous researchers had demonstrated an ability of ligands with imidazoline-like structures to bind to MAO with nanomolar affinity, at a site distinct from the active site and also to inhibit enzyme activity (although at concentrations rather higher than those required to bind to the high affinity site $)^{[4,34,41]}$. These observations suggested that mammalian amine oxidases from different sub-subfamilies may possess a characteristic allosteric regulatory site. Accordingly, we proceeded to examine the effects of a number of imidazoline ligands versus BPAO.

In initial examinations of oxidation of a single concentration of $\mathrm{BZ}$ by a range of concentrations of several imidazoline ligands, we obtained remarkable data with several ligands indicating hormetic activation of enzyme activity, compared with control assays ${ }^{[14]}$. The imidazoline ligands clonidine, cirazoline and oxymetazoline all enhanced turnover of BZ, with inhibition occurring at higher imidazoline concentrations, while efaroxan and 2-(2-benzofuranyl)2-imidazoline (2-BFI) caused partial inhibition which reached a plateau when approximately $50 \%$ of $\mathrm{BZ}$ oxidation had been inhibited. These unexpected observations were initially interpreted as suggesting the presence of an imidazoline binding site distinct from the BPAO active site, since it seemed obvious that ligands could not enhance enzyme activity through binding to the active site. Furthermore, at least some of the effects of the imidazoline ligands appeared to be allosteric, since the hormetic effects of cirazoline were substrate-dependent; only oxidation of BZ was enhanced, while turnover of other substrates was inhibited in a uniphasic or biphasic manner ${ }^{[14]}$ (Fig. 1).

Stimulation and subsequent inhibition of BPAO activity were seen to occur as a result of distinct effects on $\mathrm{K}_{\mathrm{M}}$ and $\mathrm{V}_{\max }$, implicating the involvement of more

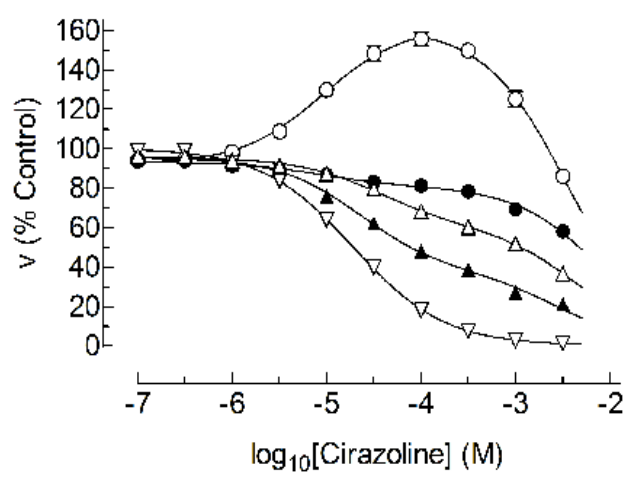

Fig. 1: The effects of cirazoline $(0.1 \mu \mathrm{M}$ to $3 \mathrm{mM})$ on oxidation by BPAO of BZ $(300 \mu \mathrm{M} ; \bigcirc)$, ptyramine $(135 \mu \mathrm{M} ; \mathbf{\Delta})$, PEA $(275 \mu \mathrm{M} ; \triangle)$, MA (420 $\mu \mathrm{M}$; $)$ and SPD $(50 \mu \mathrm{M} ; \nabla)$, concentrations approximating $50 \%$ of their respective $K_{M}$ values. From reference ${ }^{[12]}$. 
than one binding site for the imidazoline ligands ${ }^{[14]}$. Although the locations of these sites were unknown, kinetic modeling generated a hypercubic reaction scheme which implicated four distinct binding sites for cirazoline. Kinetic modeling was simplified by applying Michaelis-Menten principles to control data at low substrate concentrations where no substrate inhibition or other hormetic behaviour was clearly apparent. Nevertheless, it seemed most unlikely that four imidazoline binding sites could be present on a single enzyme monomer; rather, two sites on two forms of the enzyme, or perhaps on two monomers within a cooperative SSAO dimer, offered a more feasible explanation for the kinetic deductions.

Redox reactions catalysed by SSAO (and BPAO) may be represented by a reductive (i) and oxidative (ii) half-reaction:

- $\mathrm{E}_{\mathrm{ox}}+\mathrm{RCH}_{2} \mathrm{NH}_{3}{ }^{+}+\mathrm{H}_{2} \mathrm{O} \leftrightarrow \mathrm{E}_{\mathrm{ox}}-\mathrm{NH}^{+} \mathrm{CH}_{2} \mathrm{R} \rightarrow \mathrm{E}_{\text {red }}+$ $\mathrm{RCHO}+\mathrm{H}_{2} \mathrm{O}$

- $\mathrm{E}_{\mathrm{red}}+\mathrm{O}_{2} \rightarrow \mathrm{E}_{\mathrm{ox}}+\mathrm{H}_{2} \mathrm{O}_{2}+\mathrm{NH}_{4}^{+}$

Reduced enzyme $\left(\mathrm{E}_{\mathrm{red}}\right)$, in which the SSAO quinone cofactor has accepted the amino group from amine substrate, may exist in several forms, such as an aminoquinol/semiquinone or as an iminoquinone ${ }^{[36,48]}$. Data from the laboratories of Klinman ${ }^{[32,36]}$ and Knowles ${ }^{[48]}$ suggested that small cations, as well as substrates which would be expected to exist to some degree in cationic form at physiological $\mathrm{pH}$, could bind to reduced forms of SSAO enzymes from H. polymorpha and E. coli. Furthermore, Di Paolo had described competitive inhibition of BPAO by metal cations from groups I and II ${ }^{[7]}$, albeit over low substrate concentration ranges. We therefore investigated the abilities of the group I alkali metal cations $\mathrm{Li}^{+}, \mathrm{Na}^{+}, \mathrm{K}^{+}$, $\mathrm{Rb}^{+}$and $\mathrm{Cs}^{+}$, as well as $\mathrm{NH}_{4}{ }^{+}$, to influence activities of BPAO and human recombinant vascular wall SSAO over extended substrate concentration ranges ${ }^{[12]}$. Figure 2a shows inhibition of human $\mathrm{SSAO}$ by $\mathrm{NH}_{4}{ }^{+}$, with methylamine (MA) as substrate, while Fig. 2b shows inhibition of BPAO by $\mathrm{NH}_{4}{ }^{+}$, with spermidine (SPD) as substrate. It is clear that as the concentration of ammonium increases, the hormetic nature of substrate oxidation tends towards classical hyperbolic enzyme behaviour, suggesting that ammonium competes for the site on these enzymes through which binding of substrates causes substrate inhibition of SSAO activity. The effects of metal cations on BPAO activity were perhaps of greater significance. Figure $3 \mathrm{a}$ and $b$ show effects of $\mathrm{Li}^{+}$and $\mathrm{Cs}^{+}$on SPD oxidation by $\mathrm{BPAO}$, respectively. In addition to a loss of hormetic substrate oxidation as metal cation concentrations
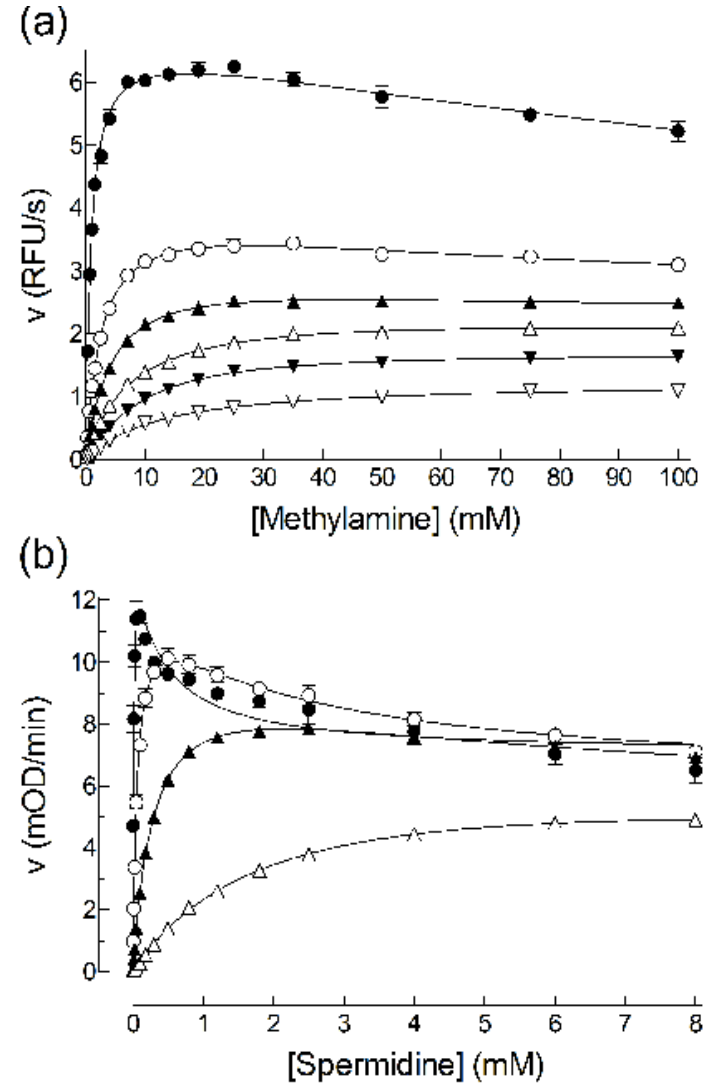

Fig. 2: (a): Effects of ammonium $(22 \bigcirc, 82 \bigcirc, 172 \triangle$, $372 \boldsymbol{\Delta}, 722 \nabla$ and $1272 \mathrm{mM} \boldsymbol{\nabla})$ on MA oxidation by human SSAO, measured in a fluorescence-based peroxidase-coupled assay, (b): Effects of ammonium on SPD oxidation by BPAO, measured in an absorbance-based peroxidase-coupled assay. Concentrations are as indicated for Fig. 2a. Experimental details are provided in reference ${ }^{[10]}$

increase, it is evident that over certain substrate concentration ranges, the effects of cations on enzyme activity are themselves hormetic (i.e., Fig. 3c). These observations suggest that an enhancement of SSAO activity may be achieved through antagonism of substrate inhibition, rather than by enzyme activation per se.

Rather than implicating a second substrate binding site through which substrate inhibition was mediated, Ockham's razor favoured an explanation involving binding of substrate to $\mathrm{E}_{\mathrm{red}}$, leading to partial or complete inhibition of enzyme activity. In this regard, SSAO enzymes may behave in a manner somewhat similar to that described for MAO enzymes, which may bind substrate at oxidised and reduced enzyme 
Am. J. Pharm. \& Toxicol., 3 (1): 125-136, 2008

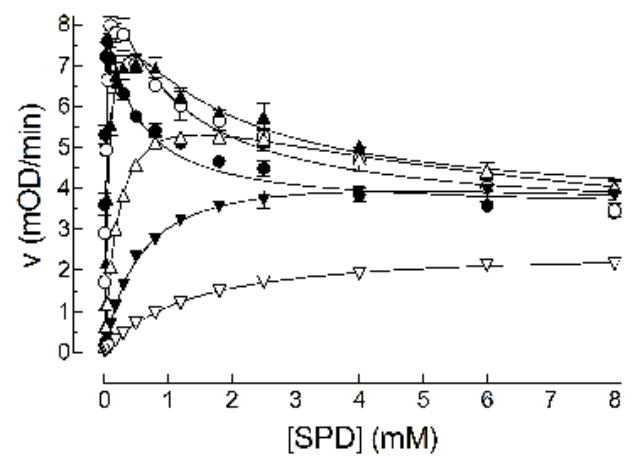

(a)

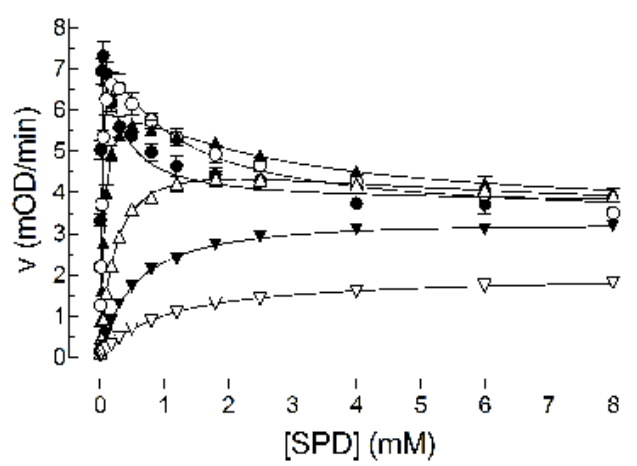

(b)

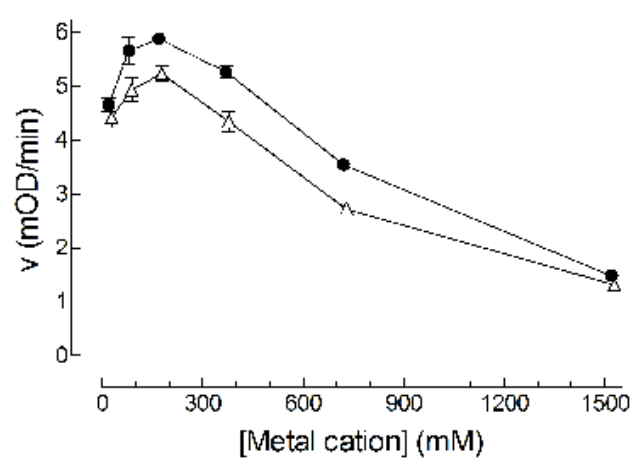

(c)

Fig. 3: (a): Effects of $\mathrm{Li}^{+}(19 \bigcirc, 79 \bullet, 169 \triangle, 369 \boldsymbol{\Delta}$, $719 \nabla$ and $1269 \mathrm{mM} \nabla$ ) on SPD oxidation by bovine plasma SSAO, measured in an absorbance-based peroxidase-coupled assay. From reference ${ }^{[10]}$. (b): Effects of $\mathrm{Cs}^{+}(28 \bigcirc, 88$ $\bullet, 178 \triangle, 378 \boldsymbol{\Delta}, 728 \nabla$ and $1528 \mathrm{mM} \boldsymbol{\nabla}$ ) on SPD oxidation by bovine plasma SSAO, measured in an absorbance-based peroxidasecoupled assay. Experimental details are provided in reference $\mathrm{e}^{[10]}$. (c): Replot of rate data in the presence of $\mathrm{Li}^{+}(\bullet)$ and $\mathrm{Cs}^{+}(\triangle)$ with a representative SPD concentration of $1.8 \mathrm{mM}$. forms ${ }^{[16]}$. Depending upon the form of the cofactor species present in the form of $\mathrm{E}_{\mathrm{red}}$ bound by substrate, such a hypothesis would require that reoxidation of reduced SSAO (aminoquinol) to yield $\mathrm{H}_{2} \mathrm{O}_{2}$, hydrolysis of the resulting iminoquinone to generate ammonium and active enzyme, or both steps, should occur more slowly, or not at all, when substrate was occupying the active site of $\mathrm{E}_{\mathrm{red}}$. It seems unlikely that this phenomenon is due simply to a reduced affinity for dioxygen, since the concentration of oxygen in airsaturated buffer is very much higher than the $\mathrm{K}_{\mathrm{M}}$ of oxygen for these enzymes ${ }^{[24]}$. In order that enzyme activity be enhanced in the presence of metal cations or an imidazoline ligand such as cirazoline, reoxidation and/or hydrolysis of $\mathrm{E}_{\text {red }}$ would have to occur more quickly with either of these ligands bound within the active site than would occur when substrate is bound. This in turn suggests that if reoxidation and/or hydrolysis can occur with a large ligand such as cirazoline bound within the active site, then regeneration of active enzyme may also occur with substrate bound to $\mathrm{E}_{\text {red }}$-but at a rate rather lower than when the active site is unoccupied. Our kinetic data demonstrate clearly that with most substrates, maximal substrate inhibition results only in partial loss of enzyme activity and this is supported by fitting of data with a pseudoquantitative rapid equilibrium approach to an equation describing partial ordered inhibition by substrates $^{[12]}$. Results of these analyses confirm that the affinities of substrates for reduced enzyme are generally very much lower than for oxidised enzyme, in agreement with the contention of Shepard and Dooley ${ }^{[44]}$, although our observations of substrate inhibition with large molecules such as SPD and spermine might argue against the presence of more than one substrate molecule within the SSAO active site at high substrate concentrations ${ }^{[44,48]}$. The crystal structure of BPAO with clonidine bound within the active site revealed that binding of clonidine was to an enzyme form in which the quinone cofactor was rotated to the on-copper (catalytically inactive) position. Rotation of the cofactor, which has been proposed to occur when the iminoquinone is deprotonated ${ }^{[36]}$, may present a more favourable environment for substrate amine binding within an otherwise unwelcoming active site and this may represent another viable route towards substrate inhibition by a single substrate molecule.

Based on results from the kinetic analyses described above ${ }^{[12]}$, as well as from previous modeling studies which had indicated that cirazoline and related compounds likely bound to four sites on $\mathrm{BPAO}^{[14]}$, we hypothesised that both substrates and imidazoline 
Am. J. Pharm. \& Toxicol., 3 (1): 125-136, 2008

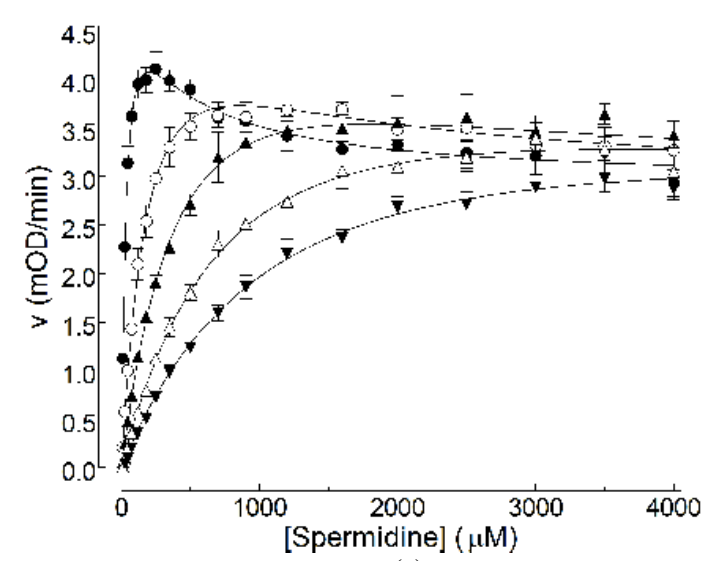

(a)

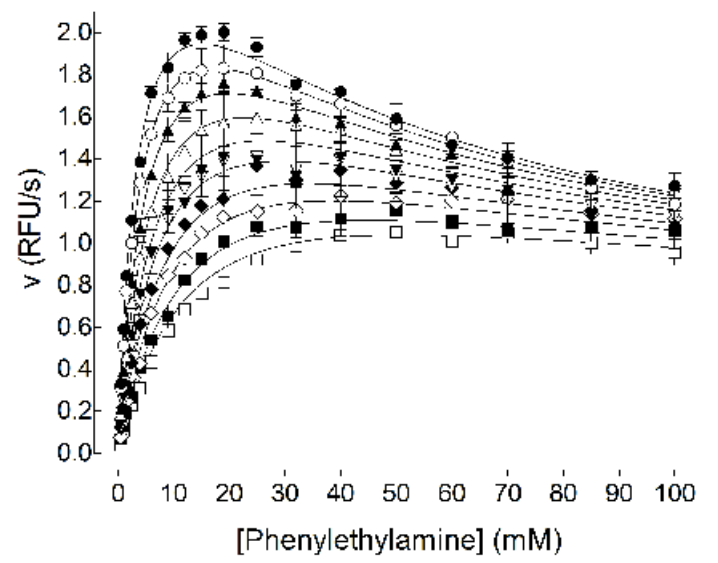

(b)

Fig. 4: (a): Effects of 2-BFI $(0 \bullet, 50 \bigcirc, 120 \boldsymbol{\Delta}, 250 \triangle$ and $400 \mu \mathrm{M} \boldsymbol{\nabla})$ on SPD oxidation by BPAO. Experimental details are provided in reference ${ }^{[11]}$. (b): Effects of guanabenz $(0-75 \mu \mathrm{M})$ on oxidation of PEA by human SSAO. From reference ${ }^{[11]}$

ligands could bind to the active site and to a secondary site recognised by tetraphenylphosphonium ${ }^{[6]}$ on both oxidised and reduced forms of SSAO enzymes. Further kinetic experiments, in conjunction with anaerobic spectroscopic studies, radioligand binding studies and X-ray crystallographic analyses of pure BPAO crystals prepared in the presence of clonidine, confirmed that this was indeed the case, at least for the bovine enzyme $^{[13]}$. No secondary site could be implicated when human SSAO was examined in parallel studies, although kinetic data were still consistent with binding of substrates and imidazoline ligands to the active site in both oxidised and reduced enzyme. Furthermore, global analyses of data obtained in the presence of imidazoline ligands such as clonidine and guanabenz were consistent with the effects of these ligands being mediated through binding to one (human) or two (bovine) sites on oxidised and reduced enzymes, in competition with substrate ${ }^{[13]}$. The results of these competitive interactions are seen clearly as a loss of hormetic behaviour coupled with a loss of substrate inhibition when SPD oxidation by BPAO is assayed in the presence of 2-BFI (Fig. 4a), or when PEA oxidation by human SSAO is assayed in the presence of guanabenz (Fig. 4b). Activation beyond control activity of the bovine enzyme, but not the human enzyme, is evident in these examples.

Unpublished analyses of theoretical data sets with equations derived to describe SSAO behaviours in the presence of substrates and imidazoline ligands ${ }^{[13]}$ suggest that an enhancement of enzyme activity is usually observed only when the overall rate of enzyme reoxidation and hydrolysis is faster in the presence of the modulator ligand than in the presence of substrate. In addition, the modulator should generally have similar or higher affinity for the oxidised enzyme, compared with the substrate, as well as a higher affinity for $E_{\text {red }}$ than for $\mathrm{E}_{\mathrm{ox}}$. These predictions are generalisations and we have observed several exceptions in our theoretical assessments. Nevertheless, while we have thus far failed to observe enhancement of human SSAO activity with the small number of ligands examined, theoretical analyses indicate that a molecule possessing the above properties would enhance activity of the human enzyme through antagonism of substrate inhibition.

\section{ANOMALOUS BEHAVIOUR OF MAO (EC 1.4.3.4) ENZYMES: PRELIMINARY OBSERVATIONS}

More recently, we have turned our attention to examinations of classical MAO enzymes, focusing initially upon human MAO-B. While much debate still surrounds the molecular mechanisms of catalysis, the general reaction scheme followed by substrates is thought to occur by one or both of two potential pathways, as described by Singer and colleagues ${ }^{[16]}$. In the case of PEA, substrate is believed to form a binary complex with enzyme, from which imine is released prior to binding of dioxygen and reoxidation of enzyme. In contrast, BZ may form a ternary complex, where binding of dioxygen and reoxidation of the enzyme occurs prior to release of imine. Against this background, we have recently observed apparent partial substrate inhibition of human MAO-B expressed on bacterial membranes, with PEA and nonylamine as 
Am. J. Pharm. \& Toxicol., 3 (1): 125-136, 2008

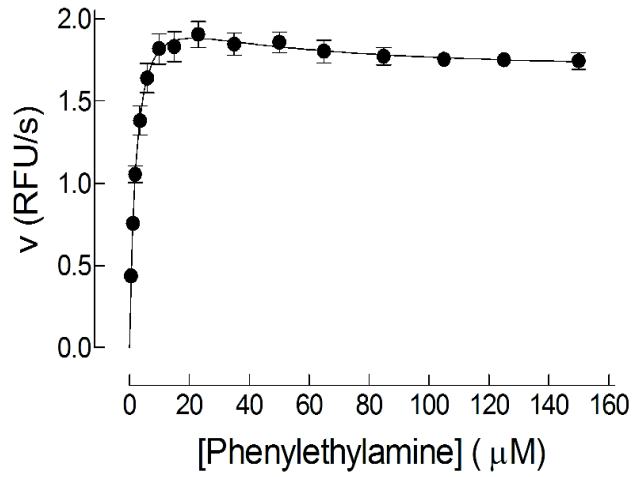

(a)

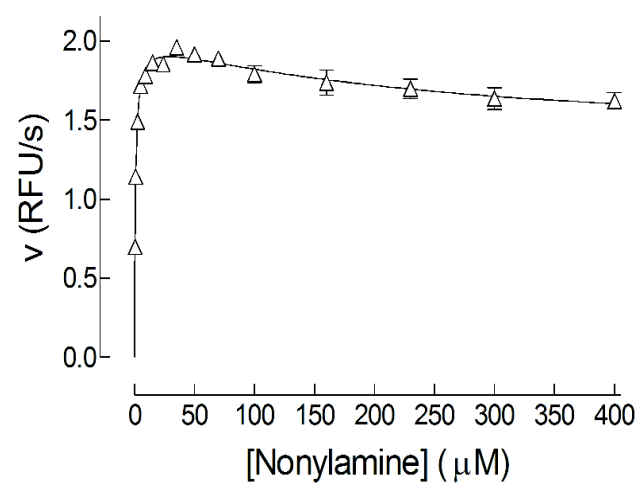

(b)

Fig. 5: Oxidation of PEA (a) and nonylamine (b) by pure human MAO-B under air reveals substrate inhibition of initial rates. Assays were done with a fluorescence-based peroxidase-coupled assay similar to that described for SSAO in reference ${ }^{[11]}$. $\mathrm{V}_{\max }$ values determined from curve-fitting to an equation describing partial ordered inhibition by substrate are 2.343 and $2.025 \mathrm{RFU} / \mathrm{s}$, respectively, illustrating the degree of underestimation that would occur through fitting of early data points to the Michaelis-Menten equation

substrates (Fig. 5). Interestingly, substrate inhibition is less apparent when pure (soluble) MAO-B is used.

It is likely that the hormetic effects observed with PEA occur, at least in part, due to an as-yet uncharacterised product inhibition, the effects of which become apparent very rapidly ${ }^{[21]}$. Product inhibition aside, the binary/ternary pathways described above can not account for this hormesis. Substrates generating a binary complex with enzyme prior to reoxidation would yield $E_{\text {red }}$ with a vacant active site; thereafter, ordered binding of substrate and dioxygen to $\mathrm{E}_{\text {red }}$, creating a ternary species, followed by reoxidation of enzyme in a

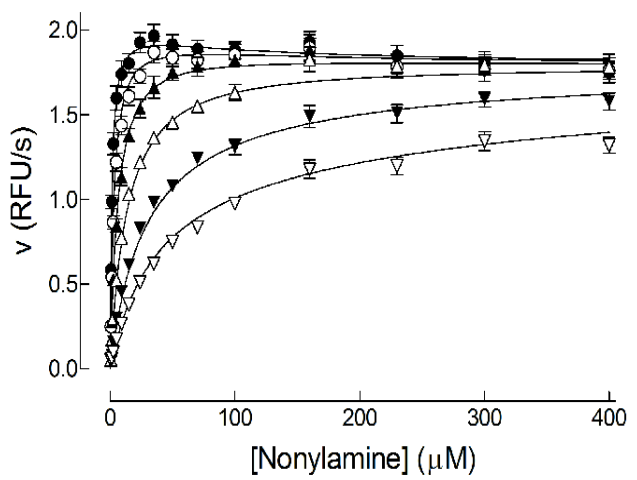

Fig. 6: Inhibition by 2-BFI of nonylamine oxidation by pure human MAO-B. Assays were done under air with a fluorescence-based peroxidasecoupled assay by a procedure identical to that described for SSAO in reference 11, in the presence of 2-BFI $(0 \bullet, 3 \bigcirc, 10 \boldsymbol{\Delta}, 30 \triangle$, $100 \boldsymbol{\nabla}$ and $300 \mu \mathrm{M} \nabla$ ). Data are fitted to a pseudoquantitative equation (see reference ${ }^{[10]}$ ) describing binding of nonylamine and 2-BFI to both oxidised and reduced enzyme forms.

manner analogous to the process thought to occur with substrate BZ, would result in a binary complex between substrate and $\mathrm{E}_{\mathrm{ox}}$ which is primed for catalytic activity. This process was suggested to occur by Ramsay and colleagues $^{[39]}$, who obtained stopped-flow data consistent with this pathway. In order for such a shortcut to be observed as substrate inhibition, reoxidation of substrate-bound enzyme would presumably occur more slowly than reoxidation of vacant enzyme, analogous to the process which we propose occurs with SSAO enzymes. However, while binding of MAO-B substrates to $\mathrm{E}_{\mathrm{red}}$ does indeed appear to alter reoxidation rates in a manner dependent upon the identity of the substrate, these changes are usually observed as enhanced rates ${ }^{[39,47]}$. Therefore, it is possible that an increase in $\mathrm{K}_{\mathrm{M}}$ for oxygen may also have occurred that might explain the observed reduction in activity, and the relatively high $\mathrm{K}_{\mathrm{M}}$ values for dioxygen observed with MAO-B demand that changes in oxygen affinity must be taken into consideration when interpreting steady-state data from kinetic experiments such as those presented here.

While substrates, but not inhibitors, were able to influence the rate of reoxidation of reduced enzyme ${ }^{[38]}$, we have nevertheless obtained preliminary kinetic data suggesting that some non-substrate ligands may bind to $\mathrm{E}_{\mathrm{red}}$ (as well as to $\mathrm{E}_{\mathrm{ox}}$ ) and influence enzyme activity, presumably as a result of competition with substrate for binding to one or both sites. These observations are consistent with historical data ${ }^{[35,40]}$ which revealed that 
several drugs, including (+) amphetamine and amitriptyline, could inhibit oxidation of several MAO$\mathrm{B}$ substrates in a manner consistent with binding both to $\mathrm{E}_{\mathrm{ox}}$ and to $\mathrm{E}_{\mathrm{red}}$. For example, Figure 6 shows effects of 2-BFI on human MAO-B-mediated oxidation of nonylamine, fitted with an equation which requires binding of substrate and 2-BFI to both $\mathrm{E}_{\mathrm{ox}}$ and $\mathrm{E}_{\mathrm{red}}$. These observations are remarkably similar to those we have made with SSAO, although experiments with MAO are at an early stage and no novel mechanisms may yet be deduced from these preliminary data. However, in contrast with (+) amphetamine and amitriptyline, the inhibitory effects of 2-BFI may be mediated in part through binding to a site distinct from the active site; in that regard, the mechanisms under study in our laboratory appear somewhat novel.

\section{DRUGS TARGETING THE REDUCED FORMS OF SSAO AND MAO AS NOVEL THERAPEUTIC AGENTS}

To date, amine oxidase inhibitors have been used experimentally and therapeutically based on an underlying assumption that inhibition results from a competitive interaction at the active site of $\mathrm{E}_{\mathrm{ox}}$. With which enzyme form irreversible inhibitors interact is largely irrelevant, since an irreversibly-inhibited enzyme plays no further part in regulation of substrate. Reversible inhibitors such as the MAO-A inhibitor moclobemide offer several pharmacokinetic and therapeutic advantages over irreversible compounds, particularly with regard to control of the extent and duration of the drug's effect. However, unless reversible inhibitors have remarkably high affinity for the enzyme, relatively large doses of drug must be administered to overcome increased competition which occurs as a result of elevated substrate concentration, even when such drugs have favourable pharmacokinetic profiles. Also, drugs targeting $\mathrm{E}_{\mathrm{ox}}$ competitively will inhibit turnover of all endogenous (or ingested) substrates, even though the desired therapeutic effect may result from inhibiting oxidation of only one amine.

The observation that amine substrates for SSAO and MAO can interact with $\mathrm{E}_{\mathrm{ox}}$, resulting in substrate oxidation and with $\mathrm{E}_{\mathrm{red}}$, resulting in a reduction or an enhancement in substrate oxidation rate, and the fact that these interactions occur with differing affinities, in a manner dependent upon the identity of the substrate and in the case of MAO at least, in a manner dependent upon the concentration of dissolved oxygen, open up the possibility of developing reversible drugs that inhibit or enhance amine oxidation in a substratespecific manner. The effects that a ligand has with respect to a particular substrate would depend upon the relative affinities of the substrate itself for $E_{o x}$ and $E_{r e d}$, as well as the observed effect on substrate oxidation (inhibition or stimulation) and the degree of that effect, following binding of substrate to $E_{\text {red }}$. The affinities of a particular ligand for $E_{o x}$ and $E_{\text {red }}$, relative to the affinities of multiple substrates for $E_{\text {ox }}$ and $E_{\text {red }}$, might then be exploited to cause substrate-specific effects. This is exemplified beautifully in Figure 1; introduction of cirazoline $(100 \mu \mathrm{M})$ into a system where BZ, SPD and MA were being oxidised simultaneously by BPAO would result in a marked enhancement of BZ oxidation and a substantial inhibition of SPD oxidation while leaving MA oxidation largely unaltered.

We may speculate upon the potential therapeutic benefits of this novel dimension of selectivity by considering putative antidepressant drugs which could inhibit oxidation of noradrenaline and serotonin by MAO-A while leaving oxidation of dietary tyramine largely unaltered. Most current SSAO inhibitors, including those in development, are irreversible in their action, blocking turnover of all endogenous SSAO substrates. As we begin to understand more about the roles of $\mathrm{H}_{2} \mathrm{O}_{2}$ as a SSAO-generated signaling molecule and about the endogenous substrates from which $\mathrm{H}_{2} \mathrm{O}_{2}$ is derived, it is likely that some benefit will be seen, for example, in inhibiting turnover of the amine which generates $\mathrm{H}_{2} \mathrm{O}_{2}$ in the lymphocyte adhesion process, without preventing $\mathrm{H}_{2} \mathrm{O}_{2}$-mediated glucose uptake into adipocytes. Indeed, an ability to enhance substrate turnover may lead to novel insulinomimetic drugs which stimulate glucose uptake into adipocytes without stimulating other SSAO-dependent peroxide-mediated signaling pathways. In all of these examples, the fact that some drug effects result from ligand binding to $\mathrm{E}_{\mathrm{red}}$, an enzyme form for which SSAO substrates have low affinity, suggests that dosing issues related to competition between drugs and substrates may be minimised, reducing the likelihood of adverse reactions occurring at higher drug doses. The possibilities seem endless and there seems no reason why this novel approach to development of enzyme modulators may not be extended to many of the other enzymes which have been shown to display hormetic behaviour towards their endogenous substrates.

\section{ACKNOWLEDGEMENT}

Research in the author's (A.H.) laboratory is funded by the Canadian Institutes for Health Research (Operating Grant MOP 77529).

We thank Owen Degenhardt for technical assistance and Drs. David Dooley, Eric Shepard and Peter Knowles for helpful discussions. 


\section{REFERENCES}

1. Akiri, G., E. Sabo, H. Dafni, Z. Vadasz, Y. Kartvelishvily, N. Gan, O. Kessler, T. Cohen, M. Resnick, M. Neeman and G. Neufeld, 2003. Lysyl oxidase-related protein-1 promotes tumor fibrosis and tumor progression in vivo. Cancer Res., 63: 1657-1666.

2. Bardsley, W.G., P. Leff, J. Kavanagh and R.D. Waight, 1980. Deviations from MichaelisMenten kinetics. The possibility of complicated curves for simple kinetic schemes and the computer fitting of experimental data for acetylcholinesterase, acid phosphatase, adenosine deaminase, arylsulphatase, benzylamine oxidase, chymotrypsin, fumarase, galactose dehydrogenase, beta-galactosidase, lactate dehydrogenase, peroxidase and xanthine oxidase. Biochem. J., 187: 739-765.

3. Barrand M.A., B.A. Callingham and S.A. Fox, 1984. Amine oxidase activities in brown adipose tissue of the rat: Identification of semicarbazidesensitive (clorgyline-resistant) activity at the fat cell membrane. J. Pharm. Pharmacol., 36: 652-658.

4. Carpéné, C., P. Collon, A. Remaury, A. Cordi, A. Hudson, D. Nutt and M. Lafontan, 1995. Inhibition of amine oxidase activity by derivatives that recognize imidazoline I2 sites. J. Pharmacol. Exp. Ther., 272: 681-688.

5. Conklin, D.J., H.R. Cowley, R.J. Wiechmann, G.H. Johnson, M.B. Trent and P.J. Boor, 2004. Vasoactive effects of methylamine in isolated human blood vessels: Role of semicarbazidesensitive amine oxidase, formaldehyde and hydrogen peroxide. Am. J. Physiol. Heart Circ. Physiol., 286: H667-H676.

6. Di Paolo, M.L., M. Lunelli, M. Scarpa and A. Rigo, 2004. Phosphonium compounds as new and specific inhibitors of bovine serum amine oxidase. Biochem. J., 384: 551-558.

7. Di Paolo, M.L., M. Scarpa, A. Corazza, R. Stevanato and A. Rigo, 2002. Binding of cations of group IA and IIA to bovine serum amine oxidase: Effect on the activity. Biophys. J., 83: 2231-2239.

8. Elsworth, J.D. and R.H. Roth, 1993. Deprenyl: Pharmacological aspects of its clinical effects. Biogenic. Amines, 9: 381-394.

9. Gahl, W.A., A.M. Vale and H.C. Pitot, 1982. Spermidine oxidase in human pregnancy serum. Probable identity with diamine oxidase. Biochem. J., 201: 161-166.
10. Haefely, W., W.P. Burkard, A. Cesura, A. Colzi, R. Kettler, H.P. Lorez, J.R. Martin, J.L. Moreau, J.G. Richards and R. Schaffner et al., 1993. Pharmacology of moclobemide. Clin. Neuropharmacol., 16: S8-S18.

11. Holt, A. and M.M. Palcic, 2006. A peroxidasecoupled continuous absorbance plate-reader assay for flavin monoamine oxidases, copper-containing amine oxidases and related enzymes. Nature Protocols, 1: 2498-2505.

12. Holt, A., O.S. Degenhardt, P.D. Berry, J.S. Kapty, S. Mithani, D.J. Smith and M.L. Di Paolo, 2007. The effects of buffer cations on interactions between mammalian copper-containing amine oxidases and their substrates. J. Neural Transm., 114: 733-741.

13. Holt, A., D.J. Smith, L. Cendron, G. Zanotti, A. Rigo and M.L. Di Paolo, 2007. Multiple binding sites for substrates and modulators of semicarbazide-sensitive amine oxidases: Kinetic consequences. Mol. Pharmacol., 73: 525-538.

14. Holt, A., B. Wieland and G.B. Baker, 2004. Allosteric modulation of semicarbazide-sensitive amine oxidase activities in vitro by imidazoline receptor ligands. Br. J. Pharmacol., 143: 495-507.

15. Hubálek, F., C. Binda, A. Khalil, M. Li, A. Mattevi, N. Castagnoli and D.E. Edmondson, 2005. Demonstration of isoleucine 199 as a structural determinant for the selective inhibition of human monoamine oxidase $\mathrm{B}$ by specific reversible inhibitors. J. Biol. Chem., 280: 15761-15766.

16. Husain M., D.E. Edmondson and T.P. Singer, 1982. Kinetic studies on the catalytic mechanism of liver monoamine oxidase. Biochemistry, 21: 595-600.

17. Ignesti, G., 2003. Equations of substrate-inhibition kinetics applied to pig kidney diamine oxidase (DAO, E.C. 1.4.3.6). J. Enzyme Inhib. Med. Chem., 18: 463-473.

18. Jalkanen, S., M. Karikoski, N. Mercier, K. Koskinen, T. Henttinen, K. Elima, K. Salmivirta and M. Salmi, 2007. The oxidase activity of vascular adhesion protein-1 (VAP-1) induces endothelial E-and P-selectins and leukocyte binding. Blood, 110: 1864-1870.

19. Kelly, I.D., P.F. Knowles, K.D. Yadav, W.G. Bardsley, P. Leff and R.D. Waight, 1981. Steady-state kinetic studies on benzylamine oxidase from pig plasma. Eur. J. Biochem., 114: 133-138. 
20. Kennedy, S.H., A. Holt and G.B. Baker, 2004. Monoamine Oxidase Inhibitors. In: Handbook of Experimental Pharmacology. Antidepressants: Past, Present and Future, Preskorn, S.H., C. Stanga, J.P. Feighner and R. Ross (Eds.). Springer-Werlag, Heidelberg, pp: 209-240.

21. Kinemuchi, H., Y. Arai, L. Oreland, K.F. Tipton and C.J. Fowler, 1982. Time-dependent inhibition of monoamine oxidase by $\beta$-phenethylamine. Biochem. Pharmacol., 31: 959-964.

22. Kragten, E., I. Lalande, K. Zimmermann, S. Roggo, P. Schindler, D. Muller, J. van Oostrum, P. Waldmeier and P. Furst, 1998. Glyceraldehyde-3-phosphate dehydrogenase, the putative target of the antiapoptotic compounds CGP 3466 and R-(-)-deprenyl. J. Biol. Chem., 273: 5821-5828.

23. Li, M., C. Binda, A. Mattevi and D.E. Edmondson, 2006. Functional role of the aromatic cage in human monoamine oxidase B: Structures and catalytic properties of Tyr435 mutant proteins. Biochemistry, 45: 4775-4784.

24. Lizcano, J.M., K.F. Tipton and M. Unzeta, 2000. Time-dependent activation of the Semicarbazide-Sensitive Amine Oxidase (SSAO) from ox lung microsomes. Biochem. J., 351: 789-794.

25. Lucero, H.A. and H.M. Kagan, 2006. Lysyl oxidase: An oxidative enzyme and effector of cell function. Cell Mol. Life Sci., 63: 2304-2316.

26. Lyles, G.A. and B.A. Callingham, 1975. Evidence for a clorgyline-resistant monoamine metabolizing activity in the rat heart. J. Pharm. Pharmacol., 27: 682-691.

27. Lyles, G.A. and I. Singh, 1985. Vascular smooth muscle cells: A major source of the semicarbazidesensitive amine oxidase of the rat aorta. J. Pharm. Pharmacol., 37: 637-643.

28. Maintz, L. and N. Novak, 2007. Histamine and histamine intolerance. Am. J. Clin. Nutr., 85: 1185-1196.

29. McEwen, C.M., 1965. Human Plasma Monoamine Oxidase. Kinetic Studies. J. Biol. Chem., 240: 2011-2018.

30. Mu, D., K.F. Medzihradszky, G.W. Adams, P. Mayer, W.M. Hines, A.L. Burlingame, A.J. Smith, D. Cai and J.P. Klinman, 1994. Primary structures for a mammalian cellular and serum copper amine oxidase. J. Biol. Chem., 269: 9926-9932.
31. Mure, M., D.E. Brown, C. Saysell, M.S. Rogers, C.M. Wilmot, C.R. Kurtis, M.J. McPherson, S.E. Phillips, P.F. Knowles and D.M. Dooley, 2005. Role of the interactions between the active site base and the substrate Schiff base in amine oxidase catalysis. Evidence from structural and spectroscopic studies of the 2-hydrazinopyridine adduct of Escherichia coli amine oxidase. Biochemistry, 44: 1568-1582.

32. Mure, M., S.A. Mills and J.P. Klinman, 2002. Catalytic mechanism of the topa quinone containing copper amine oxidases. Biochemistry, 41: 9269-9278.

33. O'Rourke, A.M., E.Y. Wang, A. Miller, E.M. Podar, K. Scheyhing, L. Huang, C. Kessler, H. Gao, H.T. Ton-Nu, M.T. Macdonald, D.S. Jones and M.D. Linnik, 2008. Anti-inflammatory effects of LJP $1586 \quad$ [Z-3-Fluoro-2-(4methoxybenzyl)allylamine hydrochloride], an amine-based inhibitor of semicarbazide-sensitive amine oxidase activity. J. Pharmacol. Exp. Ther. 324: 867-875.

34. Ozaita, A., G. Olmos, M.A. Boronat, J.M. Lizcano, M. Unzeta and J.A. Garcia-Sevilla, 1997. Inhibition of monoamine oxidase $\mathrm{A}$ and $\mathrm{B}$ activities by imidazol(ine)/guanidine drugs, nature of the interaction and distinction from I2imidazoline receptors in rat liver. $\mathrm{Br}$. J. Pharmacol., 121: 901-912.

35. Pearce, L.B. and J.A. Roth, 1985. Human brain monoamine oxidase type B: Mechanism of deamination as probed by steady-state methods. Biochemistry, 24: 1821-1826.

36. Plastino, J., E.L. Green, J. Sanders-Loehr and J.P. Klinman, 1999. An unexpected role for the active site base in cofactor orientation and flexibility in the copper amine oxidase from Hansenula polymorpha. Biochemistry, 38: 8204-8216.

37. Ramsay, R.R., 1991. Kinetic mechanism of monoamine oxidase A. Biochemistry, 30: 4624-4629.

38. Ramsay, R.R., 1998. Substrate regulation of monoamine oxidases. J. Neural Transm. Suppl., 52: 139-147.

39. Ramsay, R.R., S.C. Koerber and T.P. Singer, 1987. Stopped-flow studies on the mechanism of oxidation of N-methyl-4-phenyltetrahydropyridine by bovine liver monoamine oxidase $\mathrm{B}$. Biochemistry, 26: 3045-3050. 
40. Roth, J.A., 1979. Effect of Drugs on Inhibition of Oxidized and Reduced form of MAO. In: Monoamine Oxidase: Structure, Function and Altered Functions, Singer, T.P., R.W. von Korff and D.L. Murphy (Eds.). Academic Press, pp: 153-168.

41. Remaury, A., R. Raddatz, C. Ordener, S. Savic, J.C. Shih, K. Chen, I. Seif, E. De Maeyer, S.M. Lanier and A. Parini, 2000. Analysis of the pharmacological and molecular heterogeneity of I(2)-imidazoline-binding proteins using monoamine oxidase-deficient mouse models. Mol. Pharmacol., 58: 1085-1090.

42. Salmi, M. and S. Jalkanen, 2001. VAP-1: An adhesin and an enzyme. Trends Immunol., 22: 211-216.

43. Seiler, N., 1995. Polyamine Oxidase, Properties and Functions. In: Progress in Brain Research, Yu, P.M., K.F. Tipton and A.A. Boulton (Eds.). Elsevier Science BV, pp: 333-344.

44. Shepard, E.M. and D.M. Dooley, 2006. Intramolecular electron transfer rate between active-site copper and TPQ in Arthrobacter globiformis amine oxidase. J. Biol. Inorg. Chem., 11: 1039-1048.

45. Shou, M., R. Dai, D. Cui, K.R. Korzekwa, T.A. Baillie and T.H. Rushmore, 2001. A kinetic model for the metabolic interaction of two substrates at the active site of cytochrome P450 3A4. J. Biol. Chem., 276: 2256-2262.
46. Shou, M., Y. Lin, P. Lu, C. Tang, Q. Mei, D. Cui, W. Tang, J.S. Ngui, C.C. Lin, R. Singh, B.K. Wong, J.A. Yergey, J.H. Lin, P.G. Pearson, T.A. Baillie, A.D. Rodrigues and T.H. Rushmore, 2001. Enzyme kinetics of cytochrome P450mediated reactions. Curr. Drug Metab., 2: 17-36.

47. Tan, A.K. and R.R. Ramsay, 1993. SubstrateSpecific Enhancement of the Oxidative HalfReaction of Monoamine Oxidase. Biochemistry, 32: 2137-2143.

48. Wilmot, C.M., J. Hajdu, M.J. McPherson, P.F. Knowles and S.E. Phillips, 1999. Visualization of dioxygen bound to copper during enzyme catalysis. Science, 286: 1724-1728.

49. Youdim, M.B. and Y.S. Bakhle, 2006. Monoamine oxidase: Isoforms and inhibitors in Parkinson's disease and depressive illness. Br. J. Pharmacol., 147: S287-S296.

50. Yu, P.H., M. Wang, H. Fan, Y. Deng and D. Gubisne-Haberle, 2004. Involvement of SSAOmediated deamination in adipose glucose transport and weight gain in obese diabetic KKAy mice. Am. J. Physiol. Endocrinol. Metab., 286: E634-E641.

51. Zorzano, A., A. Abella, L. Marti, C. Carpéné, M. Palacin and X. Testar, 2003. Semicarbazidesensitive amine oxidase activity exerts insulin-like effects on glucose metabolism and insulinsignaling pathways in adipose cells. Biochim. Biophys. Acta, 1647: 3-9. 